\title{
Performance Analysis of AODV Based Maximum Energy Level Routing in Ad-Hoc Networks
}

\author{
P.S.Karadge ${ }^{1}$, Prof.Dr.Mrs.S.V.Sankpal ${ }^{2}$ \\ Department of Electronics and Telecommunication,Shivaji university,Kolhapur \\ 1punamatu@gmail.com, ㄹ. Sankpal16@yahoo.com
}

\begin{abstract}
Advances in wireless communications have enabled the development of networks of a large number of small inexpensive, low-power multifunctional devices. These wireless Adhoc networks present a very interesting and challenging area and have tremendous potential applications. Communication protocols are the heart and soul of any communicationnetwork and the same is true for the Adhoc networks. Because of their great potential for providing safer and healthier environments for human beings they have attracted extensive interest from industry and academia. These Adhoc networks are usually operated in energy constraint environments. Therefore, from a networking perspective, energy efficiency is a critical objective in the design of communication protocols. This paper describes improvement of an energy efficiency routing for ad hoc network utilizing the high energy paths. We present a comparative analysis of existing AODV protocol and energy efficient AODV protocol which is modified to improve network lifetime in MANET.Simulation results indicate that the routing schemes are more efficient than existing well-known routing protocol.
\end{abstract}

\section{Keywords}

Ad Hoc networks, Energy efficient Routing , Power Consumption ,Residual energy.

\section{Council for Innovative Research}

Peer Review Research Publishing System

Journal: International Journal of Management \& Information Technology

Vol. 6, No. 1

editor@cirworld.com

www.cirworld.com, member.cirworld.com 


\section{INTRODUCTION}

With the advance of wireless communication technologies, small-size and high-performance computing and communication devices like commercial laptops and personal digital assistants are increasingly used in daily life. After the success of second generation mobile systems, more interest was started in wireless communications. This interest has led to two types of wireless networks: infrastructure wireless network and infrastructure less wireless network, it is also called Mobile Ad-Hoc Network (MANET). Mobile Ad hoc Network is anautonomous system of mobile nodes connected bywireless links. Each node operates as an endsystem and a router for all other nodes in thenetwork. MANET is a selfconfiguring network of mobile routers connectedby wireless links -the union of which forms anarbitrary topology.Dynamic topology, unstable links, limited energy capacity and absence of fixed infrastructure are special features for MANET when compared to wired networks. MANET do not have centralized controllers, which makes it different from traditional wireless networks (cellular networks and wireless LAN) [1].MANETs, find applications in several areas. Some of them are: military applications, collaborative and distributed computing, emergency operations, wireless mesh networks, wireless sensor network, and hybrid wireless network architectures [2]. The nodes in the MANET are typically powered by batteries which have limited energy resource. Sometimes it becomes very difficult to recharge or replace the battery of nodes; in such situation energy conservations are essential. The lifetime of the nodes show strong dependence on the lifetime of the batteries. In the MANET nodes depend on each other to relay packets. The loss of some nodes may cause significant topological changes, undermine the network operation, and affect the lifetime of the network. Hence the energy consumption becomes an important issue in MANET.

In recent years, a number of studies have been done on the different layers of the OSI model, such as MAC layer and application layer, to achieve energy conservation. Our work focuses only on the routing/network layer. The shortest path is the most common criteria adopted by the conventional routing protocols proposed in the MANET working Group[3]. The problem is that nodes along shortest paths may be used more often and exhaust their batteries faster. The consequence is that the network may become disconnected leaving disparity in the energy, and eventually disconnected sub networks. Therefore, the shortest path is not the most suitable metric to be adopted by a routing decision. Other metrics that take the power constraint into consideration for choosing the appropriate route are more useful in some scenarios. The reactive routing protocol Ad hoc On-demand Distance Vector (AODV) is found to be the most energy efficient. Our work is mainly concentrated towards improving the existing AODV algorithm, using residual energy as metric, to obtain an energy efficient AODV algorithm.Proposed routing protocol is called as MELAODV that is Maximum Energy Level Ad Hoc Distance Vector (MELAODV) which introduces maximum energy path on the network layer, combining all link nodes overall remaining energy as an important parameter for route selection, the route with highest combined energy will be a better path for routing over the ad hoc network as the life span of the selected path can be extended to ensure maximum path availability for communication. In this work, we give comparative analysis of two routing protocols; Ad-hoc on demand distance vector(AODV) and The Maximum Energy Level Ad Hoc Distance Vector (MELAODV). Simulation results are obtainedusing two parameters: 1) Network lifetime 2) Depleted nodes.

\section{BACKGROUND}

Due to the limited energy resources, there has been research on improving the energy efficiency of wireless ad hoc network. Several ad hoc routing algorithms such as Dynamic Source Routing (DSR), Ad-hoc On-Demand Distance Vector Routing (AODV), Temporally-Ordered Routing (TORA) and Destination Sequenced Distance vector (DSDV) have been evaluated in term of energy consumption [4].In this work author concentrated on the energy consumption issues of the routing protocols. This gives performance comparison of the DSR, AODV, TORA and DSDV routing protocols with respect to energy consumption, evaluating how the different approaches and algorithms affect the energy usage in the mobile devices.Link quality is a metric that is used by Signal stability based adaptive routing (SSA) [5] to select best link quality route among many different routes. In addition to link quality, SSA also uses location stability as metric. Ad hoc networks require a highly adaptive routing scheme to deal with the frequent topology changes. The proposed Signal Stability-Based Adaptive Routing (SSA) protocol is novel in its use of signal strength and stability of individual hosts as route selection criteria. Selecting the most stable links (i.e., those which exhibit the strongest signals for the maximum amount of time) leads to longer-lived routes and less route maintenance.Power aware localized routing [6] achieves the goal by controlling the transmit power of the communication device. Furthermore, power-aware routing is proposed to find a low cost route instead of the shortest routing path [7]. In [8], a RED-based Minimum Energy Routing (REDME) is described, which uses MAC layer buffer queue length as an indicator of the degree of congestion. AODV-RSS (AODV with received Signal Strength) [9] uses the received signal strength and changing rate to find a route that can sustain longer. Cross based Power aware cross layer design is based on intermediate nodes to judge its ability to forward the RREQ packets or drop it [10]. Other routing algorithms also prefer the low cost route can be found in [11] [12].

\subsection{Ad hoc On-demand Distance Vector(AODV)}

AODV[13] is a on demand routing protocol which do not maintain the network topology information. They obtain the necessary path when it is required, by using a connection establishment process. Hence these protocols do not exchange routing information periodically.AODV is basically a combination of Dynamic source routing (DSR)[14] and DestinationSequenced Distance-Vector (DSDV) [15] algorithms. It uses the advantageous feature of both these algorithm. Dynamic, self-starting and multi-hop routing is allowed between participating mobile nodes. The basic on demand routing mechanism of route discovery and route maintenance of DSR and the use of hop by hop routing sequencing number and periodic update packets of DSDV are both available in AODV.It follow two phases: 
1.Route Discovery :When a source node needs to send a packet to a destination node for which it has norouting information in its table, the Route Discovery process is initiated. The source nodebroadcasts a route request (RREQ) to its neighbors. Each node that forwards the RREQpacket creates a reverse route for itself back to source node. Every node maintains twoseparate counters: a node sequence numberand a broadcast id. Broadcast id isincremented when the source issues a new RREQ. Together with the source's address, ituniquely identifies a RREQ. In addition to the source node's IP address, current sequencenumber and broadcast ID, the RREQ also contains the most recent sequence number forthe destination which the source node is aware ofnode receiving the RREQ may unicast a route reply (RREP) to the source if it is eitherthe destination or if it has a route to the destination with corresponding sequence numbergreater than or equal to that contained in the RREQ. Otherwise, it re-broadcasts theRREQ. Each node that participates in forwarding a RREP packet back to the source ofRREQ creates a forward route to the source node. Each node remembers only the nexthop unlike source routing which keeps track of the entire route. Nodes keep track of theRREQ's source IP address and broadcast ID. If they receive a RREQ packet that theyhave already processed, they discard the RREQ and do not forward it.As the RREP propagates back to the source, nodes set up forward pointers to thedestination. Once the source node receives the RREP, it may begin to forward datapackets to the destination. At any time a node receives a RREP (for any existingdestination in its routing table) containing a greater sequence number or the samesequence number with a smaller hop count, it may update its routing information for thatdestination and begin using the better route.

2.Route maintenance: If an upstream node in an active route senses a break inthe active route, it can reinitiate the route discovery procedure to establish a new route tothe destination (local route repair) or it can propagate an unsolicited route error packet (RERR) with a freshsequence number and infinity hop count to all active upstream neighbors. Those nodessubsequently relay that message to their active neighbors. This process continues until allactive source nodes are notified. Upon receiving notification of a broken link, sourcenodes can restart the discovery process if they still require the destination. Link failurecan be detected by using Hello messages or by using link-layer acknowledgements

\subsection{Difficiency of AODV}

The weakness of AODV algorithm to optimize routing with lowest delay is without consideration of power usage. The mobile communications devices in ad hoc network are batteries operated and have limited energy, so the network is an energy-constrained system. From the perspective of energy, the shortest path is not always the optimal path. If the same paths are being utilized repeatedly due to the minimum number of route, the nodes energy along these routes will be consumed quickly and may cause the topology partitioned earlier as in the classical routing protocols. The energy conservation of the network system is a key problem especially in the situation such as military areas, disaster relief,classrooms and conferences.

\section{PROPOSED ROUTING ALGORITHM (MEL-AODV)}

We propose a routing algorithm, Maximum Energy level Ad Hoc Distance Vector (MELAODV) is based on alternate maximum remaining energy routes in each node to increase the network lifetime and to achieve efficient utilization of node energy. The proposed algorithm selects the highest energy path for routing.

\subsection{MEL-AODV: Assumptions}

- The node has random movement and position.

- The node's link layer read the power informationfrom the physical interface and could reply to thelayer.

- Wireless channel is bidirectional and symmetrical, that is while the two nodes are communicating, the signalattenuation is the same.

When network nodes send and transmit packets among each other the remaining energy level of the network nodes can be extracted. Using the power consumption is not only a single criterion for deciding energy efficiency. Actually, energy efficiency can be measured by the duration of the time over which the network can maintain a certain performance level, which is usually called as the network lifetime. Hence routing to maximize the lifetime of the network is different from minimum energy routing.Minimum energy routes sometimes attract more flows, and the nodes in these routes exhaust their energy very soon; hence the whole network cannot perform any task due to the failure on these nodes. Hence, energy efficiency is not only measured by the power consumption but in more general it can be measured by the duration of time over which the network can maintain a certain performance level. The proposed routing protocol always chooses the highest energy route in route table for data transmission, thus energy consumption of nodes in the network could be effectively balanced and the average survival time of nodes in the network could be improved.

\subsection{Routing Algorithm Implementation}

The energy-saving routing algorithm ,Maximum Energy Level Ad Hoc Distance Vector protocol(MEL-AODV) has improved the RREQ handling process. MEL-AODV considers node remaining energy as a routing metric. The RREQ process for MEL-AODV is as below:

1.Path discovery process is initiated whenever a source node needs to communicate with another node for which it has no routing information in its table. 
2.Every node maintains two separate counters: a node sequence number and a broadcast id. In this routing, we maintain intermediate nodes receive one RREQ and discard other RREQ.

3. However we allow destination nodes to accept more than one route request. Meanwhile it obtains current node energy information and adds to accumulated energy field route request packet This process is continuing until destination node receive $R R E Q$ packet or intermediate nodes have route information towards destination.

4.Upon receiving first RREQ packet, the node will calculate and update accumulated energy field on destination node route table. If there are better energy route received, destination node will continue update its route table. This process continue until 2 to 3 route requests before destination node send route reply packet (RREP) to source node.

5.Then node unicast RREP to the source node reversely after getting route information with satisfying condition.

6.After receiving RREP, the source can send data through the highest energy route.

\section{The RREQ Packet of MEL-AODV contains following fields:}

- Type: type of packet that is route request packet.

- Accenergy: it is accumulative energy field which contains energy information of node.

- Hop count: indicates number of hops from the source to the current node.

- Broadcast id: ID uniquely identifying particular RREQ when taken.

- Destination IP address: IP address of destination for which route is desired.

- Destination sequence number: Latest sequence number received by source for any route towards destination.

- $\quad$ Source IP address: IP address of originating node thatissued Route Request.

- Source sequence number: Current sequence number of source node.

\section{PERFORMANCE EVALUATION OF MEL-AODV}

\subsection{Simulation Environment}

The simulation results in this paper were obtained using Network Simulator 2 version 2.35 (NS2) to perform comparison between MEL-AODV protocol and AODV. NS2 is a discrete event, object oriented, simulator developed by the VINT project research group at Carnegie Mellon University [16] which includes: nodes mobility, a realistic physical layer that includes a radio propagation model, radio network interfaces and the IEEE 802.11 Medium Access Control (MAC) protocol using the Distributed Coordination Function (DCF). NS2 is one of most popular network simulator tools worldwide. The NS2 was installed under Ubantu 10.0 as a simulation platform. The transmission range is assumed to be $250 \mathrm{~m}$ and the packet size is fixed at 512 bytes. In order to have performance result, we use 10 to 50 nodes on the simulation separately.

Table 1.Simulation Parameters

\begin{tabular}{|l|l|}
\hline MAC layer Type & IEEE802.11 \\
\hline Reception queue length & 50 \\
\hline Radio propagation model & Two Ray Ground \\
\hline Transmission Power & $0.6 \mathrm{~W}$ \\
\hline Reception Power & $0.3 \mathrm{~W}$ \\
\hline Initial Energy & $3.8 \mathrm{Joule}$ \\
\hline Packet Size & 512 bytes \\
\hline Total simulation time & $10 \mathrm{~min}$ \\
\hline
\end{tabular}

\subsection{The Parameter of Energy}

In our simulation, the networks interface of the nodeuse the following formula to compute the energy consumption

Energy $=$ Power $\times$ time $(1)$

When a node is transmitting or receiving a packet, the energyconsumption of node is calculated by transmitting power orreceiving power multiply the transmitted or received time. Ttxand Trcvare transmitted and received time of packetrespectively based on formula (3).

Time $=8 \times$ Packetsize $/$ Bandwidth(3) 
So formula (4) (5) (6) and (7) are used to calculate and deducttransmission and reception energy. By multiply transmit

Power (Powertx) with transmit time (Ttx), we are able to knowthe amount of energy used for packet transmission. Then wecan summarize the remaining energy of node after thesuccessful transmission.

Energy in transmit mode, Etx $=$ Powertx $\times \operatorname{Ttx}(4)$

Remaining energy, Enode = current energy $-\operatorname{Etx}(5)$

Energy in receive mode, Ercv $=$ Powerrcv $\times \operatorname{Trcv}(6)$

Remaining energy, Enode = current energy $-\operatorname{Ercv}(7)$

On the other hand, if a node forwards a packet, the energyconsumption is equal to the sum of the receiving andretransmitting of the packet, which formula is shown in (8)

Energy in forwarding mode, $\mathrm{Ef}=\mathrm{Etx}+\operatorname{Ercv}(8)$

\section{RESULTS}

The performance of network lifetime and percentage of depleted node are shown in Figure 1 andFigure 2. First, the performance of network lifetime isevaluated which defined by the time when the network isdisconnected due to lack of possible routes between the sourceand destination. The result shows that MEL-AODVachieved better performance because it reduces the number ofrebroadcasting and thus reduces the overall energy wastage.This also concludes that the performance of network lifetimeis better by increasing the number of nodes in the networkbecause the source and destination nodes can have moreneighbors to help forwarding and receiving the packet. BothMEL-AODV and AODV have the lowest network lifetimewhen there are less than 20 nodes in the network.

In Figure 2, we defined percentage of depletednodes as number of death nodes over total number of nodesavailable in a network.In MEL-AODV maximum energy path is used where as in AODV shortest path is used for routing. Same path is used repeatedly so nodes on that path exhaust their batteries fasterso depletion of node is more in AODV than MEL AODV. The result shows that our proposedscheme again has better performance as compared to theconventional routing protocol.

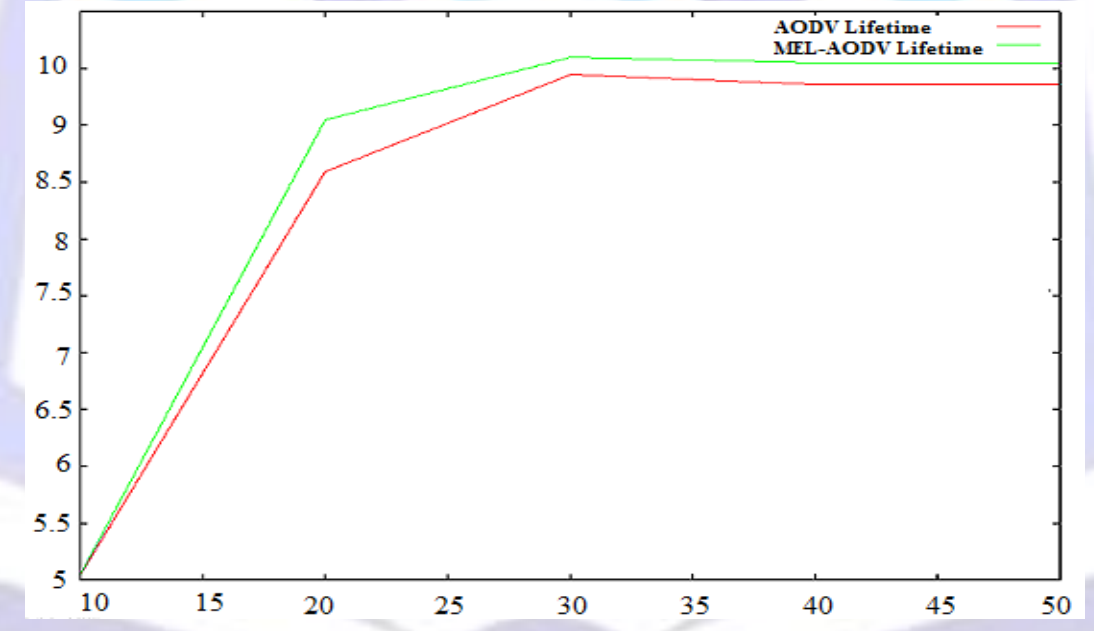

Fig.1 Network Lifetime vs number of nodes

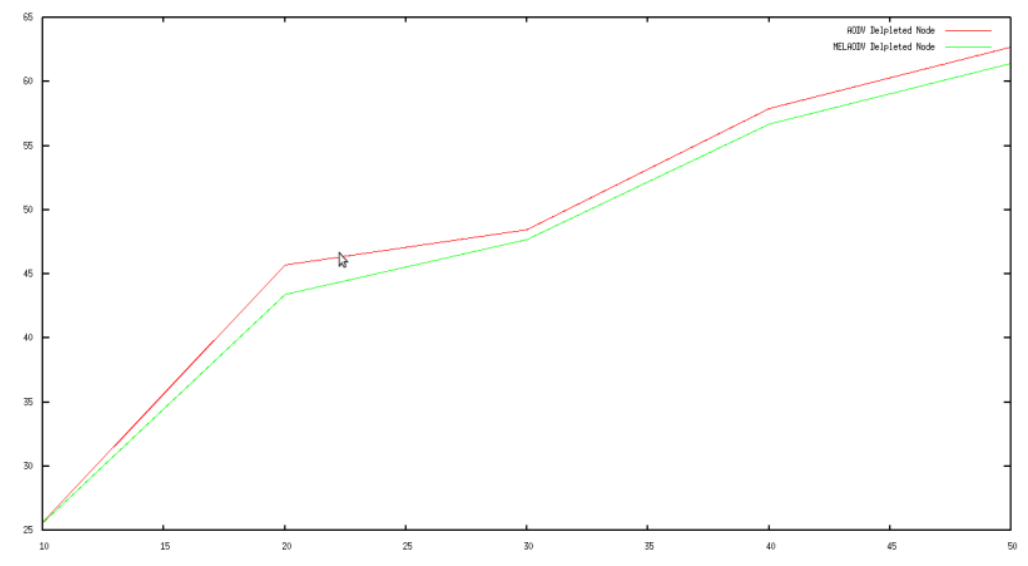

Fig.2Depleted Nodes vs number of nodes 
Table 2. Network Lifetime (sec)

\begin{tabular}{|c|c|c|}
\hline $\begin{array}{l}\text { Number of } \\
\text { nodes }\end{array}$ & MELAODV & AODV \\
\hline 10 & 5.04 & 5.04 \\
\hline 20 & 9.04 & 8.59 \\
\hline 30 & 9.6 & 9.45 \\
\hline 40 & 9.55 & 9.35 \\
\hline 50 & 9.55 & 9.35 \\
\hline
\end{tabular}

Table 3. Depleted Nodes (\%)

\begin{tabular}{|c|c|c|}
\hline $\begin{array}{l}\text { Number of } \\
\text { nodes }\end{array}$ & MELAODV & AODV \\
\hline 10 & 25.60 & 25.64 \\
\hline 20 & 43.41 & 45.71 \\
\hline 30 & 47.70 & 48.48 \\
\hline 40 & 56.71 & 57.88 \\
\hline 50 & 61.45 & 62.71 \\
\hline
\end{tabular}

\section{CONCLUSION}

The study of energy constrained routing protocols in adhoc network is attracting more attention recently. Based on themobile node remaining energy and alternate route technique, this paper presents a Maximum Energy Level AdHoc Distance Vector (MEL-AODV). From the simulationresults, the proposed scheme not only reduces energyconsumption but also prolongs the system lifetime.The proposed scheme outperforms the existing one.

\section{ACKNOWLEDGMENTS}

I would like to thank Prof S.V.Sankpal for her encouragement and comments on earlier drafts of this paper. Without the support of my loving husband it would have been impossible to complete this work.

\section{REFERENCES}

[1] S. Corson and J. Macker, January 1999. Mobile Ad hoc Networking (MANET): Issues and Evaluation Considerations, RFC 2501.

Routing Protocol Performance

[2] C. Siva Ram Murthy, B. S. Manoj, 2005. Ad Hoc Wireless Networks Architecture and Protocols, 2nd ed, Pearson Education.

[3] IETF MANET WG (Mobile Ad hoc NETwork),www.ietf.ora/html.charters/manet-charter.html

[4] Juan-Carlos Cano and Pietro Manzoni, Aug 2000 .A Performance Comparison of Energy Consumption for Mobile Ad Hoc Network Routing Protocols, Eighth International Symposium on Modeling, Analysis and Simulatio of Computer and Telecommunication Systems (MASCOTS 2000).

[5] R.Dube, C.D. Rais, K-Y Wang and S.K. Tripathi, August 28, 1996. Signal Stabiliity-based Adaptive Routing (SSA) for Ad Hoc Mobile Networks, Techical Report CSTR-3646, UMIACS-TR-96-34.

[6] Ivan Stojmenovic and Xu Lin, October 2001 .Power-aware localized routing in wireless networks, IEEE Transactions on Parallel and Distributed System, vol. 12, no.10.

[7] S,Singh, M.Woo, and C.S Raghavendra, October 1998. Power aware Routing in Mobile Ad Hoc Networks, International Conference on Mobile Computing and Neworking (Mobicom'98), pp. 181-190.

[8] X.Jin, W.Cai and Y.Zhang,2005.A RED based minimum energy routing algorithm for wireless ad-hoc networks,Proceedings of International Conference on Wireless Communications Networking and Mobile Computing, vol. 2, pp. IV757-IV761.

[9] Ruav-Shiung Chang, SInng-Jiuan Leu, Jan 2006.Long-lived Path Routing With Received Signal Strength for Ad Hoc Networks, In Proceeding of the $1^{\text {st }}$ International Symposium on Wireless Pervasive Computing. 
[10] Rekha Patil and Dr A.Damodaram, December 2008.Cost Based Power Aware Cross Layer Routing Protocol for Manet, IJCSNS International Journal of Computer Science and Network Security, vol. 8, No.12.

[11] Jae-Hwan Chang and Leandros Tassiulas, IEEE Infocom 2000.Energy Conserving Routing in Wireless Ad Hoc Networks, The Conference on Computer Communications, pp.22-31.

[12] Ram Ramanathan, Regina Rosales-Hain, IEEE Infocom 2000.Topology Control of Multihop Wireless Networks using Transmit Power Adjustment, The Conference on Communications.

[13] C. E. Perkins, E. M. Belding-Royer, and S. Das, July 2003, .Ad hoc on-demand distance vector (AODV) Routing, RFC 3561, work in progress http://www.ietf.org/rfc/rfc3561.txt

[14] David Johnson, David Maltz and Yih-Chun Hu, July 2004.The Dynamic Source Routing Protocol for Mobile Ad Hoc Networks, Internet Draft, draft-ietf-manet-dsr- 10.txt, work in progress.

[15] C. E. Perkins and P. Bhagwat, Aug 1994. Destination-Sequenced Distance Vector (DSDV) for Mobile Computers, Proc. of the SIGCOMM 1994 Conference on Communications Architectures, Protocols and Applications, pp 234-244.

[16] Kevin Fall, Kannan Varadhan. January 2008. The ns Manual (formerly ns Notes andDocumentation), the VINT Project: Collaboration between researchers at UCBerkeley, LBL, USC/ISI and Xerox PARC.

\section{Author' biography}

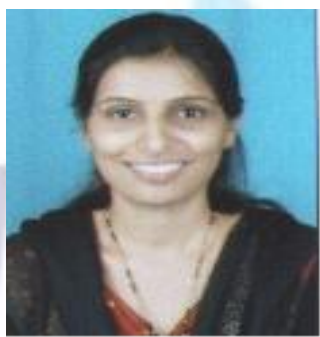

Karadge P S.

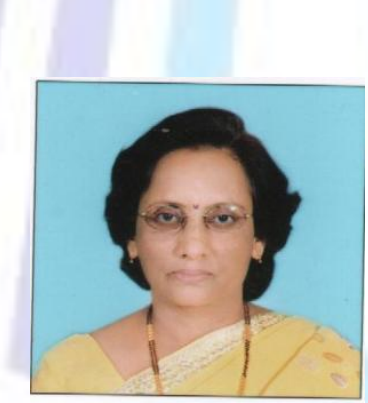

Prof.Dr.Mrs.Swati V.Sankpal
Karadge $\mathbf{P} \mathbf{S}$. is currently working towards her M.E (Electronics and telecommunication) at D. Y.Patil college of engg. and Tech, affiliated to shivaji university Kolhapur, India. She received her B.E (Elecrtonics) from Walchand college of Engg and technology Sangali, in 2007. Her research interest include Ad Hoc networks.

Prof.Dr.Mrs.Swati V.Sankpal is currently working as associate professor at D.Y.Patil college of Engg. and Tech, Kolhapur. She has total 28 years of experience.She has completed her B.E and M.E.in Electronics from Walchand College of Engg.Sangli and earned her Ph.D. from Shivaji University, Kolhapur. She has published total 25 national conference papers, 25 international conference papers, 22 international journal papers. Under her guidance six students have completed their P.G.(E.\&.TC) and, ten are pursuing. 